\title{
Differential effects of non-informative vision and visual interference on haptic spatial processing
}

\author{
Robert Volcic · Joram J. van Rheede • \\ Albert Postma · Astrid M. L. Kappers
}

Received: 12 February 2008/Accepted: 25 May 2008/Published online: 14 June 2008

(C) The Author(s) 2008

\begin{abstract}
The primary purpose of this study was to examine the effects of non-informative vision and visual interference upon haptic spatial processing, which supposedly derives from an interaction between an allocentric and egocentric reference frame. To this end, a haptic parallelity task served as baseline to determine the participantdependent biasing influence of the egocentric reference frame. As expected, large systematic participant-dependent deviations from veridicality were observed. In the second experiment we probed the effect of non-informative vision on the egocentric bias. Moreover, orienting mechanisms (gazing directions) were studied with respect to the presentation of haptic information in a specific hemispace. Non-informative vision proved to have a beneficial effect on haptic spatial processing. No effect of gazing direction or hemispace was observed. In the third experiment we investigated the effect of simultaneously presented interfering visual information on the haptic bias. Interfering visual information parametrically influenced haptic performance. The interplay of reference frames that subserves haptic spatial processing was found to be related to both the effects of non-informative vision and visual interference. These results suggest that spatial representations are influenced by direct cross-modal interactions; inter-participant differences in the haptic modality resulted in differential effects of the visual modality.
\end{abstract}

R. Volcic $(\square)$ · J. J. van Rheede · A. Postma .

A. M. L. Kappers

Helmholtz Institute, Utrecht University, Princetonplein 5,

3584 CC Utrecht, The Netherlands

e-mail: r.volcic@phys.uu.nl
Keywords Haptic - Space perception .

Reference frames · Non-informative vision .

Visual interference

\section{Introduction}

Information about the world reaches us through more than one sense. The integration of input from different sensory modalities is, therefore, an important aspect in forming a representation of objects and the surrounding environment. A single object may generate different sensory inputs across multiple sensory channels. Hence, strong interactions between the modalities shape the integrated percept of the object. However, different information can also originate from sources extrinsic to the object. For instance, information that belongs to the surrounding environment could also be relevant for perception of the object at issue. In this case, when information across modalities is not explicitly associated, the integration of information is possible but certainly not a necessary consequence. If and how these latter integration processes occur is not yet clear. To tackle these questions we evaluated the interaction between the processes involved in haptic perception of space and the effects of non-informative vision and visual interference. Haptic spatial tasks usually induce very large biases and are, therefore, especially suitable for the study of these effects.

Haptic spatial processing, as a unimodal perceptual experience, has been shown to be prone to substantial systematic deviations from veridicality (e.g., Blumenfeld 1937; Henriques and Soechting 2005; Lederman et al. 1987; von Skramlik 1934a, b). The haptic perception of space has been analyzed especially through the use of the haptic parallelity task (e.g., Kappers 1999; Kappers and Koenderink 1999). In this task blindfolded participants were 
instructed to rotate a test bar in such a way that they felt it to be parallel to a reference bar that was located at a different position. A methodical series of studies has mapped the magnitude and direction of deviations occurring at different spatial locations (Kappers 1999; Kappers and Koenderink 1999), different planes (Hermens et al. 2006; Kappers 2002; Volcic et al. 2007) and even in three dimensions (Volcic and Kappers 2008). The systematic deviations observed in this variety of experimental conditions were reliably accounted for by supposing a biasing effect of hand orientation as the origin of the error patterns (Kappers 2004, 2005; Kappers and Viergever 2006; Volcic et al. 2007). Note that the concept of parallelity is implicitly defined with respect to the environment, that is, with respect to an allocentric reference frame. Participants are thus required to transform the spatial information in this reference frame. Therefore, it was proposed that haptic spatial processing could be described as the interplay of an egocentric reference frame fixed to the perceiver's hand and an allocentric reference frame, reflecting the spatial properties of the surrounding environment. A similar hypothesis has also been sustained by numerous studies in visuomotor literature (e.g., Battaglia-Mayer et al. 2003; Carrozzo and Lacquaniti 1994; Cohen and Andersen 2002; Soechting and Flanders 1992, 1993). Interestingly, in the haptic parallelity task the same error patterns were found in both unimanual and bimanual experiments suggesting a common origin of the error patterns. An influence of an egocentric reference frame fixed to the perceiver's hand is actually not surprising; inevitably, the initial stages of haptic spatial processing are tuned to the part of the body that is directly in contact with the environment, in our case, the hand. The reference frames hypothesis was implemented in a weighted average model that balanced the contributions of an egocentric and an allocentric reference frame (Kappers 2007; Volcic and Kappers 2008). Importantly, this model could account for the variability in the magnitude of the deviations (ranging up to $90^{\circ}$ ) observed among participants (Kappers 2003). Greater deviations from veridicality shown by some participants were interpreted as the consequence of a more heavily weighted egocentric reference frame, whereas smaller deviations indicated the participants stronger reliance on the allocentric reference frame. In line with this explanation is also the effect of temporal delay in the haptic parallelity task (Zuidhoek et al. 2003). Performance improved when a delay was introduced between the perception of a reference bar and the setting of the test bar. This improvement was interpreted as a reinforcement of the contribution of the allocentric reference frame as also suggested by other studies (e.g., Rossetti and Régnier 1995; Rossetti et al. 1996).

A research area complementary to the unimodal studies of haptic spatial processing has addressed the issue of the influence of additional sources of information such as, for instance, vision. Newport et al. (2002) reported that noninformative vision modifies the performance in the haptic parallelity task. Non-informative vision was referred to as vision of the near space without any visual input that is directly relevant to the task at hand. Deviations were still systematic, but they were reduced in comparison to the condition in which no extra visual source of information was available. This effect could be interpreted within the weighted average model as the consequence of an enhancement of the influence of the allocentric reference frame. Non-informative vision supplements the available information about the environment and thus reduces the biasing influence of the egocentric reference frame, resulting in smaller deviations. A further effect studied in combination with the non-informative vision effect was ascribed to the head- and eye-orienting mechanisms (Zuidhoek et al. 2004). Orienting the gaze towards the region of space where the reference bar was located yielded smaller deviations than orienting it towards the test bar. This effect ensued independently of the non-informative vision effect. Unfortunately, the reference bar in this study was always located in the left hemispace and this might have confounded the effect of orienting direction with a hemispace effect. The question of whether orienting mechanisms improve tactile processing has been addressed especially in tactile detection and discrimination studies. The impact of the orienting mechanisms on tactile processing is, however, still undecided (e.g., Driver and Grossenbacher 1996; Honoré et al. 1989; Kennett et al. 2001; Pierson et al. 1991; Tipper et al. 1998).

Haptic spatial processing has also been recently studied in combination with interfering visual information (Kaas et al. 2007). A bar was visually presented on a screen and the participant had to simultaneously perform the haptic parallelity task. The visual bar was either in a congruent or incongruent orientation with respect to the haptic reference bar. As a result, the deviations were modulated by the degree to which the visual information of the object orientation was incongruent with the haptic information. The effect of visual interference was observed despite the fact that visual and haptic information were provided in different spatial locations and different planes. Although participants received visual input, this stimulation lacked any information about the surrounding environment and therefore no effect of non-informative vision was observed. Kaas et al. (2007) concluded that the visual input was combined with the haptic input despite the incongruence between visual and haptic information and the explicit instruction to ignore the visual information.

The main aim of this paper was to pinpoint the effects of non-informative vision and visual interference in relation to the inter-participant differences that are common in 
haptic spatial processing. The effects of both non-informative vision and visual interference require some sort of integration between haptic and visual information in order to occur. Therefore, we might hypothesize that the participant-dependent tendency in haptics to rely more either on the allocentric or on the egocentric reference frame possibly interacts with the degree to which the two effects arise. The participant-dependent reliance on a specific reference frame could either induce or prevent the visuohaptic integration. Consequently, it would result in stronger or weaker non-informative vision and visual interference effects. Those participants that are characterized by a more egocentric performance would profit more by integrating the additional visual information. The integration processes would then counterbalance the biasing influence of the egocentric reference frame and improve performance. On the other hand, their haptic egocentric bias could also be so prevailing that it would result in a stronger or weaker suppression of any visual information. Performance would then be unaffected by these additional sources. The way the allocentric and the egocentric reference frames are weighted has been shown to largely differ between participants. If we define a continuum between the reference frame fixed to the space and the egocentric reference frame, participants' performances can be located all the way along this range. One example of a clear individual difference concerns also a gender effect: males on average show a weaker bias of the egocentric reference frame than females (Kappers 2003; Zuidhoek et al. 2007). However, Kappers (2003) showed that male and female distributions of performances were highly overlapping and that the interparticipant differences were larger than the difference between genders. Therefore, a gender-based distinction might oversimplify the problem by not considering a more general gender-independent mechanism that could be at the origin of haptic spatial processing. If a relation exists between the weighting of reference frames in haptic spatial processing and the integration of visual information, we predicted a modulation of the size of the effects in relation to the participant-dependent weighting of reference frames.

In the first experiment we measure the baseline deviations in the haptic parallelity task to determine the degree to which each participant is biased by the egocentric reference frame. This task is then used in the two subsequent experiments as a tool to study the effects of non-informative vision and visual interference. In all the experiments we contrast a gender-based subdivision of the data with a description of the data that takes into account the full spectrum of inter-participant differences. In the second experiment, the addition of non-informative vision to the haptic parallelity task is presumed to improve the performance of the participants. Whether the improvement depends on the size of the biasing influence of the egocentric reference frame is one of the central questions of this paper. Besides this, our interest also concerned how the orienting mechanisms in the non-informative vision experiment interact with the presentation of haptic information in a specific hemispace. In the third experiment, the performance in the haptic spatial task was addressed in combination with interfering visual information. Incongruent visual information about the orientation of the object is supposed to parametrically bias the performance on the haptic parallelity task in the direction of the mismatch between haptic and visual inputs. The question again was whether the influence of visual interference is related to the tendency to rely more heavily on either the allocentric or egocentric frame of reference.

\section{Materials and methods}

\section{Participants}

Twenty undergraduate students (ten males and ten females) were recruited in this research and were remunerated for their efforts. Participants had normal or corrected-to-normal vision and normal haptic, somatosensory and motor functioning. None of the participants had any prior knowledge of the experimental design and the tasks. Handedness was assessed by means of a standard questionnaire (Coren 1993). One participant was left-handed, five participants could be considered ambidextrous, expressing only a slight preference for one or the other hand, and the other 13 participants were right-handed.

\section{Apparatus and stimuli}

The setup consisted of a table $(150 \times 75 \times 75 \mathrm{~cm})$ on which two iron plates $(30 \times 30 \mathrm{~cm})$ were positioned on either side of the participant's midsagittal plane. The iron plates were covered with a plastic layer on which a protractor with a radius of $10 \mathrm{~cm}$ was printed. The centers of the two protractors were $120 \mathrm{~cm}$ apart and $15 \mathrm{~cm}$ from the long table edge. The participant was seated in front of the table on a stool, which was adjusted so that the shoulders of different participants were always at the same height $(110 \mathrm{~cm})$. An aluminum bar, with an axle in the middle, was inserted in the center of each protractor and could be rotated freely. Small magnets were attached under the bar to prevent accidental rotations. Two bars with a length of $20 \mathrm{~cm}$ and a diameter of $1 \mathrm{~cm}$ were used as the test and reference bars. The bars had an arrow-shaped end on one side that allowed the reference bar orientation and the test bar orientation to be read with an accuracy of $0.5^{\circ}$. The bars were hidden from view by a wooden board $15 \mathrm{~cm}$ above the bars, and participants were covered up to the 
neck by a sheet attached to this board in order to prevent orientation cues from the orientation of their own body parts (see Fig. 1). Two additional iron plates were positioned on the top of the board exactly above the other iron plates. These iron plates were covered with black cardboard discs (radius of $22.5 \mathrm{~cm}$ ) that prevented participants from seeing the protractors and avoided a direct frame of reference for the visual stimulus. A hole in the center of the discs allowed the insertion into the iron plate of a round magnet marker (radius of $1.25 \mathrm{~cm}$ ) or of a bar depending on the experimental condition.

Four oblique reference orientations were tested, namely $22.5^{\circ}, 67.5^{\circ}, 112.5^{\circ}$ and $157.5^{\circ}$. The $0^{\circ}$ orientation was aligned along the left-right axis of the table and an increase in degrees signifies a rotation in the counterclockwise direction. The reference bar could be positioned at either
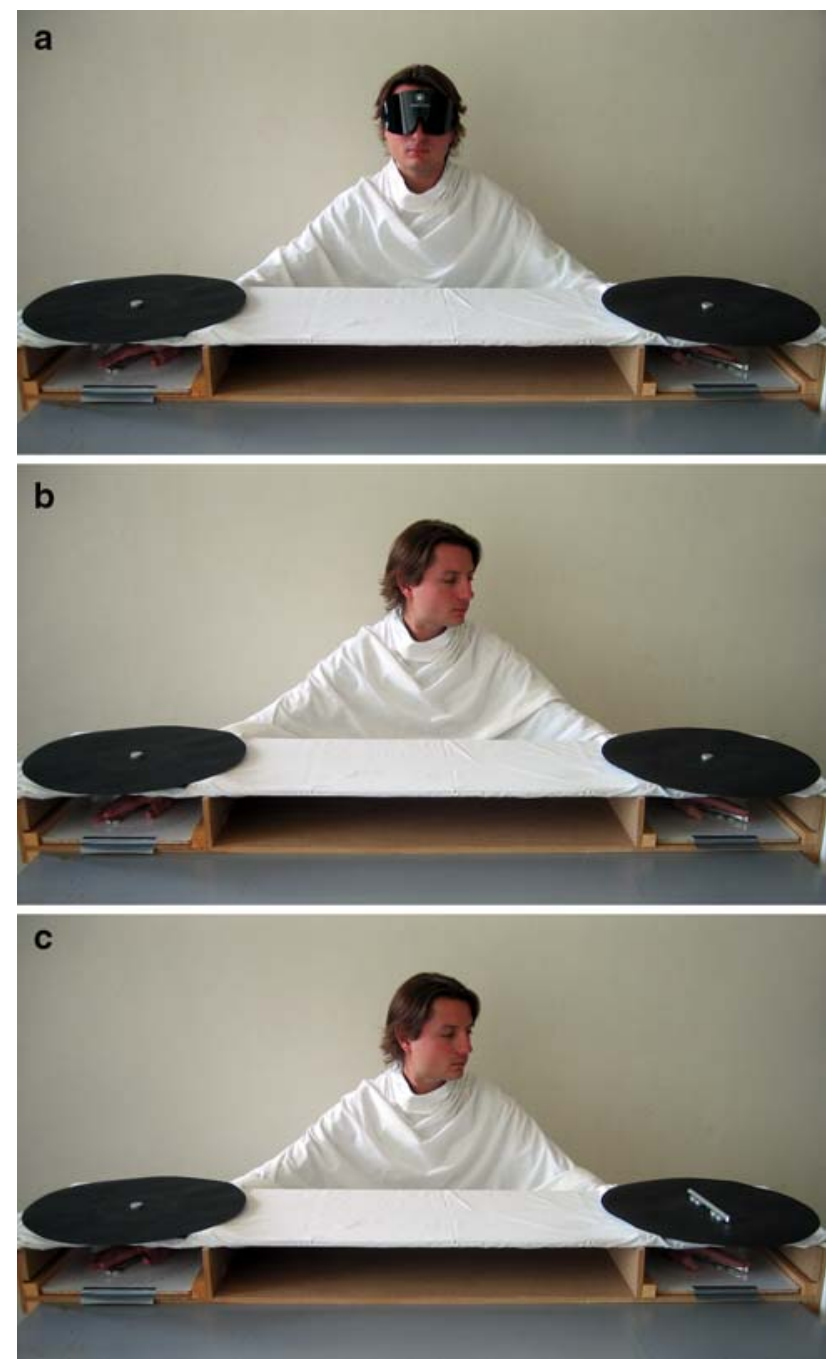

Fig. 1 Frontal views of the setup in the three experiments. a Haptic baseline experiment. b Non-informative vision experiment. c Visual interference experiment the left or the right side of the setup, that is, in the left or the right hemispace. The test bar was located on the opposite position and presented in a random orientation. Both these bars were hidden below the wooden board and were explored only by touch.

This study consists of three experiments: a Haptic baseline experiment, a Non-informative vision experiment and a Visual interference experiment. In the haptic baseline experiment, participants completed 4 reference bar orientations $\left(22.5^{\circ}, 67.5^{\circ}, 112.5^{\circ}\right.$ and $\left.157.5^{\circ}\right) \times 2$ reference bar positions (left hemispace vs. right hemispace) $\times 3$ repetitions $=24$ trials. In the non-informative vision experiment, participants completed 4 reference bar orientations $\left(22.5^{\circ}\right.$, $67.5^{\circ}, 112.5^{\circ}$ and $\left.157.5^{\circ}\right) \times 2$ reference bar positions (left hemispace vs. right hemispace) $\times 2$ gazing directions (towards either the reference or the test bar) $\times 3$ repetitions $=48$ trials. In the visual interference experiment, participants completed 4 reference bar orientations $\left(22.5^{\circ}\right.$, $67.5^{\circ}, 112.5^{\circ}$ and $\left.157.5^{\circ}\right) \times 2$ reference bar positions (left hemispace vs. right hemispace $) \times 5$ visual incongruencies $\left(-40^{\circ},-20^{\circ}, 0^{\circ}, 20^{\circ}, 40^{\circ}\right.$ compared to the haptic orientation) $\times 3$ repetitions $=120$ trials. In total, each participant completed 192 trials. The order of trials within each experiment was random and different for each participant, whereas the order of the three experiments was the same for all participants.

\section{Procedure}

Participants had to perform a bimanual parallelity task. The experimenter set the reference bar and announced to the participant which bar served as the reference bar for this trial. The participants were instructed to rotate the test bar in such a way that they felt it to be parallel to the reference bar. Both bars were touched simultaneously for the whole duration of each trial; the left hand always touched the left bar, the right hand the right bar. Participants had $10 \mathrm{~s}$ to explore the bars and orient the test bar, which appeared to be a more than adequate amount of time. An electronic digital timer measured the time, with a beep signaling when it had run out. Participants then removed their hands from the setup and after the experimenter noted down the orientation of the test bar, the next trial commenced. No feedback was given about their performance.

In the haptic baseline experiment, participants were blindfolded and asked to orient their head in alignment with the midsagittal plane (see Fig. 1a). Participants kept this posture for all the trials of this experiment. The noninformative vision experiment was essentially similar, but the blindfold was removed and participants were asked to orient their head and direct their gaze to a marker in the center of the black cardboard discs above either the reference or the test bar (see Fig. 1b). The experimenter, 
therefore, not only announced which bar would serve as the reference bar, but also in which direction participants should orient their head and direct their gaze. The visual interference experiment was similar to the latter, but instead of markers, bars identical to those used for the haptic exploration were positioned in the center of the black cardboard discs (see Fig. 1c). In this experiment, the visual bar was always positioned above the haptic reference bar. For each trial, the visual bar was set to deviate $-40^{\circ},-20^{\circ}, 0^{\circ}, 20^{\circ}$ or $40^{\circ}$ with respect to the reference bar below. Participants were told to always orient their head and direct their gaze towards the visual bar. Additionally, participants were told that the visual bar may or may not be aligned with the haptic bar below, and were explicitly asked, therefore, to use the haptic bar as a reference, as they did in the previous two experiments.

Breaks were introduced between experiments, and the visual interference experiment was interrupted by two short breaks to prevent fatigue. Participants took on average $3 \mathrm{~h}$ to complete all three experiments.

\section{Data analysis}

Previous studies using the haptic parallelity task have established that the deviations vary in a systematic way. Deviations occur in a counterclockwise direction when the reference bar is on the right of the test bar, whereas they occur in a clockwise direction when the reference bar is on the left of the test bar. Such deviations are defined as the orientation of the left bar minus the orientation of the right bar; therefore, the deviation specifies both the direction and the magnitude of the error. Positive values correspond to deviations in the expected direction, and negative values to deviations in the opposite direction. Suppose that the left bar is set at $112.5^{\circ}$ and the right bar at $70^{\circ}$, then the resulting deviation corresponds to $42.5^{\circ}$ that is in accordance with the direction found in previous studies. The orientation of the visual bar was similarly defined: a positive value corresponds to a rotation in the direction of the expected haptic deviation of the test bar, whereas a negative value corresponds to a rotation in the opposite direction. Suppose that the visual bar is set at $132.5^{\circ}$ above the previously mentioned left haptic bar, given that the rotation of the bar is in the opposite direction to the expected direction, the orientation of the visual bar is defined to be $-20^{\circ}$.

To isolate the pure effects of non-informative vision and visual interference we followed the procedures represented in Fig. 2. Specifically, the effect of noninformative vision was expressed as the percent change between the haptic baseline experiment and the noninformative vision experiment. For each participant

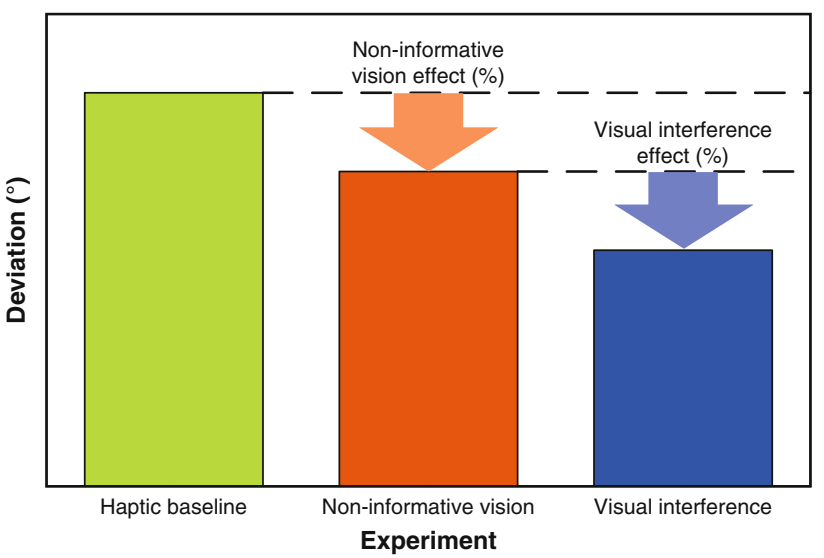

Fig. 2 Representation of the calculation of the non-informative vision effect and the visual interference effect. The non-informative vision effect was expressed as a percent change between the haptic baseline and the non-informative vision experiments. The visual interference effect was expressed as a percent change between the non-informative vision and the visual interference experiments. Note that the changes between experiments are not necessarily negative, so in fact the arrows might point upwards

individually the percent changes of the non-informative vision conditions were calculated with respect to the average deviation of the haptic baseline experiment. Therefore, a negative percent change indicates a decrease in the deviation from veridicality and conversely a positive percent change indicates an increase in the deviation from veridicality. Similarly, the effect of visual interference was expressed as the percent change between the non-informative vision experiment and the visual interference experiment. For each participant individually the percent changes of the visual interference conditions were calculated with respect to the average deviation of the non-informative vision experiment. Negative and positive percent changes indicate decreases and increases in the deviations from veridicality, respectively.

In the repeated measures analyses on the data of the visual interference experiment, the assumption of sphericity was tested, and if necessary the degrees of freedom were corrected using the Greenhouser-Geisser $\varepsilon$ correction. The minimal level of significance retained was 0.05 . In all the follow-up repeated measures analyses and in the pairwise comparisons the Holm's procedure (Holm 1979) was applied to lower the minimal level of significance. Outlier analyses were conducted on the data used in the regression analyses.

The focus of our study was mainly directed to the effects of non-informative vision and visual interference. Consequently, the reference bar orientation was not included as a statistical factor in the data analyses. Different orientations were included both to increase the variety of stimuli and to enlarge the data set. 


\section{Results}

The bar charts in Fig. 3 show the average deviations male and female participants made in the haptic baseline, the non-informative vision and the visual interference experiments. Deviations in the non-informative vision and visual interference experiments are separated for conditions. We ran a repeated measures ANOVA with experiment as the within-subject factor and gender as the between-subject factor as a crude comparison between experiments. Withinexperiment conditions were blocked. The experiment factor was significant $[F(2,36)=9.964, P<0.001]$, whereas the interaction between experiment and gender was not significant $[F(2,36)=1.67, P=0.203]$. On the other hand, the gender factor was significant $[F(1,18)=16.702$, $P<0.001]$. Subsequent pair-wise comparisons showed that performance in the haptic baseline experiment was worse than performance in both the non-informative vision $[t(19)=3.155, P<0.01]$ and visual interference experiments $[t(19)=3.513, P<0.01]$. No significant difference was found between the latter two experiments $[t(19)=$ 1.755, $P=0.098$ ]. Separate follow-up repeated measures ANOVA were conducted for the male and female groups. The factor experiment was significant for both the male and female groups $[F(2,18)=5.881, P<0.05$, and $F(2,18)=$ 5.627, $P<0.05$, respectively]. However, pair-wise comparisons showed a different pattern for the two groups. The male group performed better in the non-informative vision than in the haptic baseline experiment $[t(9)=3.349$, $P<0.05]$. No significant differences was found between the visual interference experiment and the non-informative vision experiments $[t(9)=0.484, P=0.64]$. The comparison between the haptic baseline experiment and the
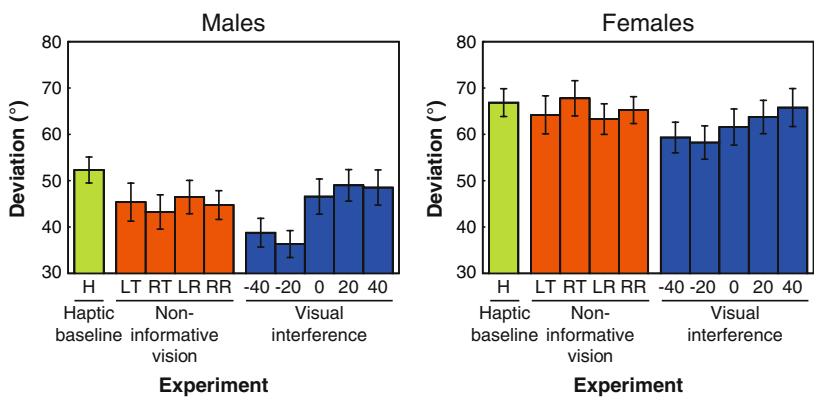

Fig. 3 The bar charts represent the deviations in the haptic baseline, the non-informative vision and the visual interference experiments. Deviations in the different conditions are shown for the latter two experiments. The left bar chart presents the male group data and the right bar chart presents the female group data. The error bars indicate the standard error of the mean. $H$ haptic, $L T$ gazing direction to the left hemispace towards the test bar, $R T$ gazing direction to the right hemispace towards the test bar, $L R$ gazing direction to the left hemispace towards the reference bar, $R R$ gazing direction to the right hemispace towards the reference bar, $-40,-20,0,20,40-$ misalignments between the haptic and visual bars visual interference experiment just failed to reach significance $[t(9)=2.504, P=0.068]$. On the other hand, the female group did not show any significant improvement in the non-informative vision experiment with respect to the haptic baseline experiment $[t(9)=1.173, P=0.271]$. The comparisons between the haptic baseline and visual interference experiments, and between the non-informative vision and visual interference experiments just failed to reach significance $[t(9)=2.65, P=0.052$, and $t(9)=$ 2.922, $P=0.051]$.

Further analyses on the single experiments with a more careful attention on the different conditions within each experiment and especially on the pure effects of noninformative vision and visual interference are presented below.

\section{Haptic baseline experiment}

The bar chart in Fig. 4 shows the distribution of deviations among participants in the haptic baseline experiment. Light-colored bars represent male participants, whereas dark-colored bars represent female participants. It is evident that all participants significantly and systematically deviated from veridicality, although the magnitude of the deviations is clearly participant-dependent. A repeated measures ANOVA with reference bar position (left hemispace vs. right hemispace) as the within-subjects factor and gender as the between-subject factor showed that there was neither an effect of reference bar position $[F(1,18)=0.299$, $P=0.591]$ nor an interaction between reference bar location and gender $[F(1,18)=0.143, P=0.709]$. On the other hand, the gender factor was significant [males, $52.3^{\circ} \pm 2.8^{\circ}$ SEM; females, $66.8^{\circ} \pm 3^{\circ}$ SEM; $F(1,18)=12.248$, $P<0.005]$; on average male participants were characterized by smaller deviations than female participants.

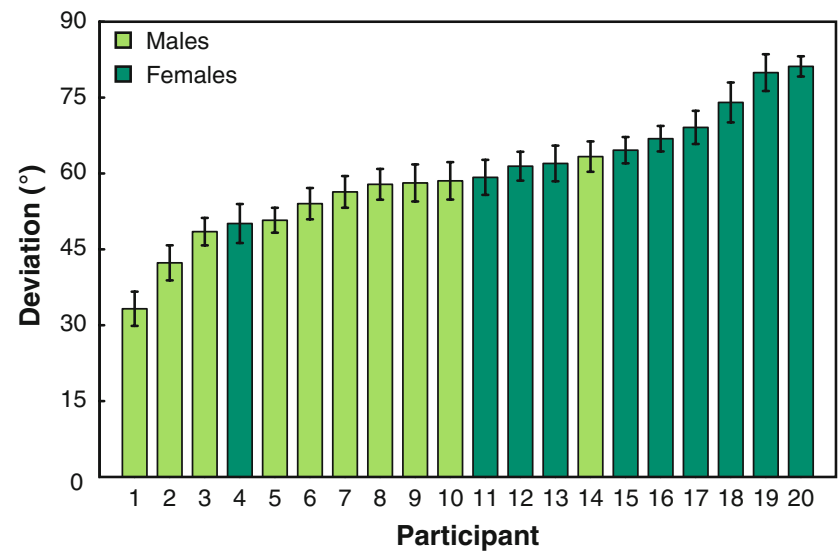

Fig. 4 Haptic baseline experiment. Bar chart of the distribution of deviations from veridicality among participants. The error bars indicate the standard error of the mean 
Non-informative vision experiment

In the non-informative vision experiment, the percent changes for the separate conditions with respect to the haptic baseline experiment are shown in Fig. 5. The factors of reference bar position (left hemispace vs. right hemispace) and gazing direction (towards either the reference or the test bar) were analyzed in a repeated measures ANOVA with gender as a between-subjects factor. The factors of reference bar position $[F(1,18)=0.139, P=0.714]$ and gazing direction $[F(1,18)=0.09, P=0.767]$ were not significant. No interaction reached significance $[0.014<F(1,18)<$ $1.816, P>0.195]$ except for the interaction between reference bar position and gender $[F(1,18)=5.055, P<0.05]$, but follow-up repeated measures ANOVAs separated by gender did not reveal any difference between hemispaces $[F(1,9)=1.837, \quad P=0.208$, and $F(1,9)=3.296, \quad P=$ 0.103 , for the male and female group, respectively]. The difference between genders was significant [males, $-14.7 \pm$ $4.7 \%$ SEM; females, $-2.7 \pm 2.4 \%$ SEM; $F(1,18)=5.06$, $P<0.05]$; on average the non-informative vision effect was smaller for females than males. Follow-up repeated measures ANOVAs on the gazing direction factor, but conducted separately on the male and female groups, did not reveal any significant effect $[0.448<F(1,9)<1.073, \quad P>0.327]$. Consequently, data were grouped among the conditions of reference bar position and gazing direction. Simple twotailed $t$ tests conducted separately on the male and female groups were run to check if non-informative vision actually decreased the magnitude of the deviations. For the male group, the non-informative vision effect was significantly different from zero $[t(9)=-3.097, P<0.05]$. On the contrary, the non-informative vision effect failed to reach significance for the female group $[t(9)=1.119, P=0.292]$.
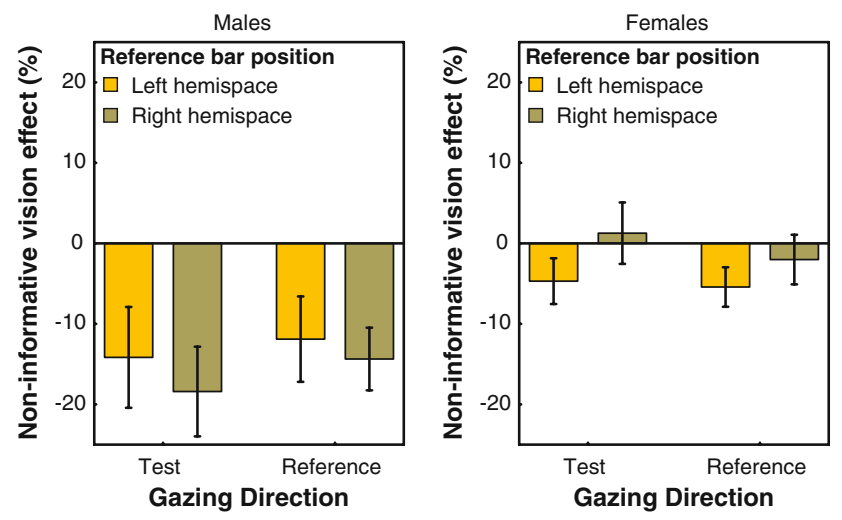

Fig. 5 Non-informative vision experiment. The bar charts represent the non-informative vision effect for the conditions of gazing direction and reference bar position. The left bar chart presents the male group data and the right bar chart presents the female group data. The error bars indicate the standard error of the mean
To further explore the non-informative vision effect we considered the magnitude of the effect as a function of the average haptic deviation and gender by conducting a stepwise regression analysis for factor selection. We decided on a significance level of 0.05 in order to determine which factors to include in the models, and a level of 0.1 to determine which to remove. The gender factor did not produce a significant improvement of the regression model; therefore, it was removed from the analysis. On the other hand, we found that the average haptic deviation was a significant predictor of the non-informative vision effect $[F(1,18)=4.507, P<0.05)$. The non-informative vision effect (percent change) could be expressed as:

$-39+0.5 \times$ average haptic deviation $(r=0.45)$.

Therefore, in the range of interest the larger the average haptic deviation was, the smaller the non-informative vision effect tended to be (see Fig. 6).

Visual interference experiment

In the visual interference experiment the within-subject factor of visual interference $\left(-40^{\circ},-20^{\circ}, 0^{\circ}, 20^{\circ}, 40^{\circ}\right.$ compared to the haptic orientation) and the betweensubject factor of gender were analyzed in a repeated measures ANOVA. Note that negative values of the visual interference are away from the expected direction of deviation, and positive values are towards the expected direction of deviation. The percent changes with respect to the non-informative vision experiment as a function of the visual interference are shown in Fig. 7. In comparison with veridicality, a positive direction indicates an increase and a negative direction a decrease in the deviations. The main effect of visual interference was significant $[F(1.865,33.571)=32.929, P<0.00001, \varepsilon=0.466]$, as

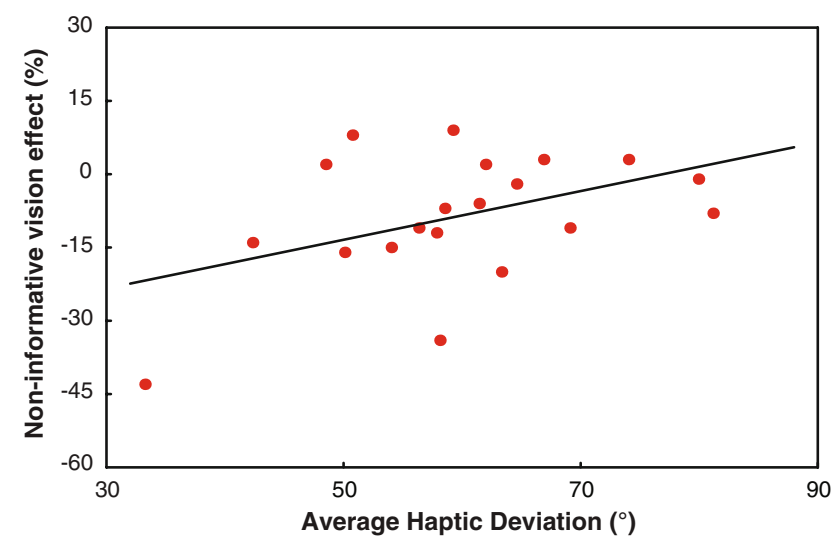

Fig. 6 Non-informative vision experiment. Scatter plot of the noninformative vision effect plotted against the average haptic deviation. Each data point represents the non-informative vision effect for a single participant 

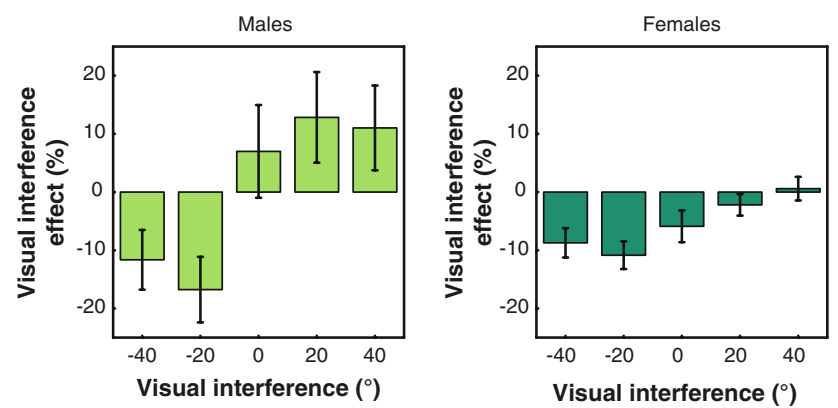

Fig. 7 Visual interference experiment. Bar chart of the visual interference effect plotted as a function of the visual interference. The left bar chart presents the male group data and the right bar chart presents the female group data. The error bars indicate the standard error of the mean

was the interaction between visual interference and gender $[F(1.865,33.571)=9.328, \quad P<0.001, \quad \varepsilon=0.466]$. On the contrary, the gender factor was not significant $[\mathrm{F}(1,18)=0.783, \quad P=0.388]$. Pair-wise comparisons showed that all the differences between visual interference levels were significant $(0.00001<P<0.05)$, except for the comparison between $-40^{\circ}$ and $-20^{\circ}$. Follow-up repeated measures ANOVAs with the same factors, but conducted separately on the male and female groups, revealed a significant main effect of visual interference for both males $[F(1.589,14.3)=25.689, \quad P<0.0001$, $\varepsilon=0.397]$ and females $[F(4,36)=8.355, P<0.0001]$. Subsequent pair-wise comparisons revealed significant differences between all visual interference levels for the male group $(2.498<t(9)<6.095,0.001<P<0.005)$, except for the comparisons between $-40^{\circ}$ and $-20^{\circ}, 0^{\circ}$ and $20^{\circ}, 0^{\circ}$ and $40^{\circ}$, and between $20^{\circ}$ and $40^{\circ}$ $[0.766<t(9)<2.779, P>0.084]$. On the contrary, significant differences were found for the female group between visual interference levels of $-20^{\circ}$ and $20^{\circ}$, and of $-20^{\circ}$ and $40^{\circ}[t(9)=3.944, P<0.05$. and $t(9)=$ 3.823, $P<0.05$, respectively]. All other comparisons did not reach significance $[1.215<t(9)<3.353, P>0.064]$.

The visual interference effect was further analyzed by linearly regressing the percent changes as a function of the visual interference for each participant individually. The slopes of the regression function were used as estimates of the strength of the visual interference effect. Since the intercepts do not convey any information of interest they were not further analyzed. Simple two-tailed $t$ tests showed that the visual interference strength was significantly different from zero for both the male group $[t(9)=5.626$, $P<0.0005]$ and the female group $[t(9)=3.542$, $P<0.01]$. Subsequently, we considered the strength of the visual interference effect as a function of the average haptic deviation and gender by conducting a stepwise regression analysis for factor selection. The same criteria as

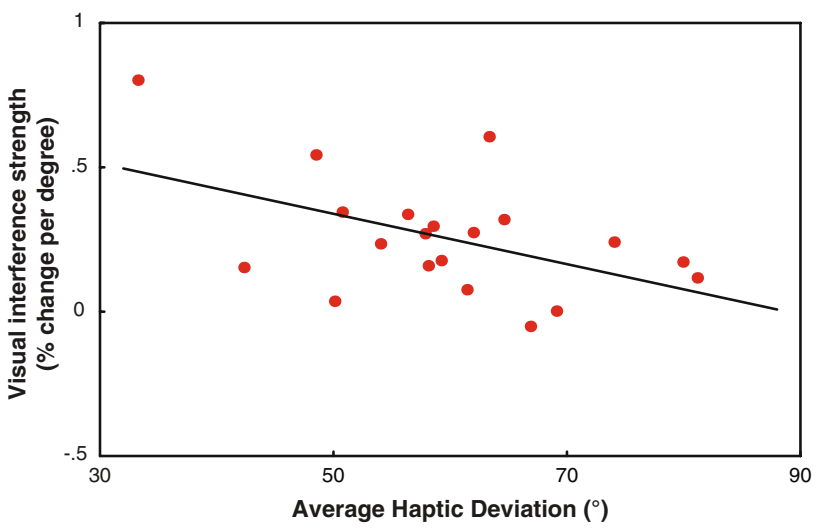

Fig. 8 Visual interference experiment. Scatter plot of the visual interference strength (slope of the regression line fitted to the individual visual interference effects as a function of the visual interference) plotted against the average haptic deviation. Each data point represents the visual interference strength for a single participant

above were used to decide which factors to include and which to exclude. Again the gender factor did not produce a significant improvement of the regression model. On the other hand, the average haptic deviation showed to be a significant predictor of the visual interference effect $[F(1,18)=5.785, \quad P<0.05]$. The visual interference strength (percent change/degree) could be expressed as:

$0.77-0.009 \times$ average haptic deviation $(r=0.49)$.

From this it follows that when the average haptic deviation was larger, the visual interference strength and therefore the visual interference effect became weaker (see Fig. 8).

\section{Discussion}

In the present study, we addressed the question of how certain aspects of the visual sensory modality interact with haptic spatial processing. We explored the connection between haptic spatial processing and the influence of noninformative vision (i.e., vision of the near space, but without any visual information that is directly relevant to the task). Furthermore, we examined the interfering effect of visual information by simultaneously providing discordant haptic and visual inputs. Our main interest was to tackle the question of whether the occurrence and the strength of the effects of non-informative vision and visual interference are modulated by the inter-participant differences usually present in haptic spatial processing, which are thought to reflect a differential contribution of an egocentric and an allocentric reference frame.

A first comparison between experiments showed that additional sources of visual information had an 
ameliorating effect on performance. However, this first simplified conclusion does not take into account the differences between the conditions of each experiment, and the expected gender-related and the inter-participant idiosyncrasies. To deepen the understanding of these factors we discuss the three experiments separately.

Our first experiment, the haptic baseline experiment, confirmed the systematic pattern of deviations found in previous studies on the parallelity task. Participants deviated substantially from veridicality showing all the same directional bias: the right bar had to be rotated clockwise with respect to the left bar in order to be perceived as parallel and vice versa. The magnitude of these deviations was observed to be participant-dependent and ranged from $33^{\circ}$ to $81^{\circ}$. Although males showed an advantage in performance with respect to females, the distributions of deviations of the two genders were not strictly separated but overlapped, suggesting common underlying processes determining the systematic deviations. The systematic directionality of the deviations provided further strong evidence that the origin of these deviations has to be linked to the biasing influence of the hand orientation. We suggest that the magnitude of the deviations depends on the proportion to which the egocentric and the allocentric frames of reference contribute. This interpretation finds support in all the previous studies on haptic parallel matching (for a review, see Postma et al. 2008). The present results make clear that the distribution of deviations in the population can range as a continuum between the representations of space defined by the allocentric and the egocentric reference frames.

In our second experiment, participants were allowed to see the surrounding environment during the haptic parallelity task. Therefore, visual information was available, but it was non-informative with respect to the demands of the task. Despite this, we observed a beneficial effect of noninformative vision on performance. Possibly, additional information on the position of bars and hands could also originate from proprioception (e.g., from neck muscles) and may contribute to this effect. The direction and the size of the effect were consistent with previous studies. Zuidhoek et al. (2004) reported an improvement of about $9 \%$, whereas Newport et al. (2002), who used a setup with different characteristics, measured an improvement of about $17 \%$. In our experiment the average improvement due to non-informative vision was $8.7 \%$. Thus, the visual information of the surrounding environment stimulates the use of the allocentric reference frame and consequently reduces the biasing effect of the egocentric reference frame. Interestingly, the difference in performance between genders increased in this experiment. Males improved by $14.7 \%$, whereas females did not significantly change their performance $(2.7 \%)$. To disentangle a purely gender based difference from a difference that originates in how haptic spatial information is processed, we considered as a further step in the analysis of this effect the magnitude of the noninformative vision effect as a function of the average haptic deviation. The average haptic deviation measured in the haptic baseline experiment was taken as the indicator of the contributions of the reference frames. We found that the average haptic deviation was a significant predictor of the non-informative vision effect. Specifically, the larger the average haptic deviation, the smaller the non-informative vision effect was in the range of interest. In this light, we can suppose that the larger the biasing influence of the egocentric reference frame, the more likely the suppression of the processes that integrate haptic and visual information will be. The reinforcement of the allocentric reference frame was, therefore, less likely to occur. This relation indicates that the preexisting tendency to recode haptic spatial information into a specific reference frame may have a direct influence on the way visual information, when made available, is integrated.

A secondary purpose of the non-informative vision experiment was to explore the relation between orienting mechanisms and hemispace. Zuidhoek et al. (2004) found that orienting the gaze towards the reference bar induced a decrease in the deviations in the haptic parallelity task. The facilitation could be explained by a more accurate perception of the orientation of the reference bar in relation to the left hand, since the reference bar was always placed in the left hemispace. Our experimental design counterbalanced both the gazing direction and the position of the reference bar in either the left or the right hemispace. Neither the gazing direction nor the hemispatial position showed a significant change in performance. The fact that no gazing direction effect was observed could be due to a difference in the experimental design: Zuidhoek et al. (2004) grouped the trials in a blocked design according to the gazing direction, whereas in our experiment the trials were completely randomized. We might speculate that the occurrence of the gazing direction effect is dependent on a prolonged allocation of attention to the position in space where the relevant stimulus is located.

In our third experiment, the visual interference experiment, haptic performance was parametrically varied by the simultaneously presented visual information. When the visual bar was presented in an incongruent orientation with respect to the haptic bar, but in the opposite direction to the haptic systematic deviation, a partial reduction of the haptic systematic deviation was shown. An opposite pattern was observed, but less clearly, when the visual bar was presented in an incongruent orientation with respect to the haptic bar, but in the same direction as the haptic systematic deviation. Interestingly, even in the case when haptic and visual information were congruent, performance 
could still vary with respect to the non-informative vision experiment. On the basis of the foregoing, it might be hypothesized that an intrinsic misalignment between haptic and visual reference frames could be at the origin of this discrepancy. The general pattern of deviations as a function of the visual interference is in accordance to the effect reported by Kaas et al. (2007). Although the visual input was explicitly defined as irrelevant for the task at hand, we observed a partial integration between modalities that interfered with the execution of the haptic parallelity task.

The visual interference strength estimated from the slope of the fitted regression line was different among participants. The steepness of the slope was more pronounced for the group of male participants. For this reason, as a further step, we explored the visual interference strength as a function of the average haptic deviation. Similarly to the non-informative vision experiment, we found that the average haptic deviation was a significant predictor of the visual interference strength. The lower the average haptic deviation was, the more conspicuous the visual interference strength tended to be, thus indicating an inability to disregard a visual stimulus that is close and similar to the haptic stimulus. On the other hand, participants that showed a stronger hand orientation bias were less likely to be influenced by the simultaneously presented visual information. Therefore, also the way the visual interference effect occurs suggests that the mechanisms underlying haptic spatial processing can exert influence on how the information from the visual modality is processed and integrated.

A generally accepted view is that the brain employs multiple frames of reference to construct spatial representations of the external world (Colby and Duhamel 1996; Flanders and Soechting 1995; Gross and Graziano 1995; Paillard 1991). For haptic spatial processing we propose that an egocentric and an allocentric reference frame interact in the construction of the representation of space, where the biasing influence of the egocentric reference frame can vary in its magnitude between participants. This interpretation was shown to well describe the inter-participant differences. In addition, we suggest that the specific contributions of the two reference frames can promote or impede the integration of supplemental sources of visual information. Spatial processes specific to the haptic modality may influence the processes that combine haptic and visual information. These results are in support of the existence of strong but flexible cross-modal associations in the construction of spatial representations.

In summary, we showed a beneficial effect of noninformative vision and a biasing effect of interfering visual information on haptic perception of space. Most interestingly, the magnitude of the hand orientation bias was found to be related to both the effects of non-informative vision and visual interference.
Acknowledgments This research was supported by the Netherlands Organization of Scientific Research (NWO).

Open Access This article is distributed under the terms of the Creative Commons Attribution Noncommercial License which permits any noncommercial use, distribution, and reproduction in any medium, provided the original author(s) and source are credited.

\section{References}

Battaglia-Mayer A, Caminiti R, Lacquaniti F, Zago M (2003) Multiple levels of representation of reaching in the parietofrontal network. Cereb Cortex 13:1009-1022

Blumenfeld W (1937) The relationship between the optical and haptic construction of space. Acta Psychol 2:763-773

Carrozzo M, Lacquaniti F (1994) A hybrid frame of reference for visuo-manual coordination. Neuroreport 5:453-456

Cohen YE, Andersen RA (2002) A common reference frame for movement plans in the posterior parietal cortex. Nat Rev Neurosci 3:553-562

Colby CL, Duhamel J-R (1996) Spatial representations for action in parietal cortex. Cogn Brain Res 5:105-115

Coren S (1993) The left-hander syndrome. Vintage Books, New York

Driver J, Grossenbacher PG (1996) Multimodal spatial constraints on tactile selective attention. In: Inni $\mathrm{T}$, McClelland $\mathrm{JL}$ (eds) Attention and performance XVI. MIT Press, Cambridge, pp 209235

Flanders M, Soechting JF (1995) Frames of reference for hand orientation. J Cogn Neurosci 7:182-195

Gross CG, Graziano MSA (1995) Multiple representations of space in the brain. Neuroscientist 1:43-50

Henriques DYP, Soechting JF (2005) Approaches to the study of haptic sensing. J Neurophysiol 93:3036-3043

Hermens F, Kappers AML, Gielen CCAM (2006) The structure of frontoparallel haptic space is task dependent. Percept Psychophys 68:62-75

Holm S (1979) A simple sequentially rejective multiple test procedure. Scand J Stat 6:65-70

Honoré J, Bourdeaud'hui M, Sparrow L (1989) Reduction of cutaneous reaction time by directing eyes towards the source of stimulation. Neuropsychologia 27:367-371

Kaas AL, van Mier HI, Lataster J, Fingal M, Sack AT (2007) The effect of visuo-haptic congruency on haptic spatial matching. Exp Brain Res 183:75-85

Kappers AML (1999) Large systematic deviations in the haptic perception of parallelity. Perception 28:1001-1012

Kappers AML (2002) Haptic perception of parallelity in the midsagittal plane. Acta Psychol 109:25-40

Kappers AML (2003) Large systematic deviations in a bimanual parallelity task: further analysis of contributing factors. Acta Psychol 114:131-145

Kappers AML (2004) The contributions of egocentric and allocentric reference frames in haptic spatial tasks. Acta Psychol 117:333340

Kappers AML (2005) Intermediate frames of reference in haptically perceived parallelity. In: World haptics conference (WHC 2005): First joint eurohaptics conference and symposium on haptic interfaces for virtual environment and teleoperator systems. IEEE Computer Society, Los Alamitos, CA, pp 3-11

Kappers AML (2007) Haptic space processing: allocentric and egocentric reference frames. Can J Exp Psychol 61:208-218

Kappers AML, Koenderink JJ (1999) Haptic perception of spatial relations. Perception 28:781-795 
Kappers AML, Viergever RF (2006) Hand orientation is insufficiently compensated for in haptic spatial perception. Exp Brain Res 173:407-414

Kennett S, Taylor-Clarke M, Haggard P (2001) Noninformative vision improves the spatial resolution of touch in humans. Curr Biol 11:1188-1191

Lederman SJ, Klatzky RL, Collins A, Wardell J (1987) Exploring environments by hand and foot: time-based heuristics for encoding distance in movement space. J Exp Psychol Learn Mem Cogn 13:606-614

Newport R, Rabb R, Jackson SR (2002) Noninformative vision improves haptic spatial perception. Curr Biol 12:1661-1664

Paillard J (1991) Motor and representational framing of space. In: Paillard J (ed) Brain and space. Oxford University Press, New York, pp 163-182

Pierson JM, Bradshaw JL, Meyer TF, Howard MJ, Bradshaw JA (1991) Direction of gaze during vibrotactile choice reaction time tasks. Neuropsychologia 29:925-928

Postma A, Zuidhoek S, Noordzij ML, Kappers AML (2008) Keep an eye on your hands: on the role of visual mechanisms in processing of haptic space. Cogn Process 9:63-68

Rossetti Y, Régnier C (1995) Representations in action: pointing to a target with various representations. In: Bardy BG, Bootsma RJ, Guiard Y (eds) Studies in perception and action III. Laurence Erlbaum, Matwah, pp 233-236

Rossetti Y, Gaunet F, Thinus-Blanc C (1996) Early visual experience affects memorization and spatial representation of proprioceptive targets. Neuroreport 7:1219-1223
Soechting JF, Flanders M (1992) Moving in three-dimensional space: frames of reference, vectors, and coordinate systems. Annu Rev Neurosci 15:167-191

Soechting JF, Flanders M (1993) Parallel, interdependent channels for location and orientation in sensorimotor transformations for reaching and grasping. J Neurophysiol 70:1137-1150

Tipper SP, Lloyd D, Shorland B, Dancer C, Howard LA, McGlone F (1998) Vision influences tactile perception without proprioceptive orienting. Neuroreport 9:1741-1744

Volcic R, Kappers AML (2008) Allocentric and egocentric reference frames in the processing of three-dimensional haptic space. Exp Brain Res (in press)

Volcic R, Kappers AML, Koenderink JJ (2007) Haptic parallelity perception on the frontoparallel plane: the involvement of reference frames. Percept Psychophys 69:276-286

von Skramlik E (1934a) Die Grundlagen der haptischen Geometrie. Naturwissenschaften 22:601-608

von Skramlik E (1934b) Die Grundlagen der haptischen Geometrie. Naturwissenschaften 22:623-627

Zuidhoek S, Kappers AML, van der Lubbe RHJ, Postma A (2003) Delay improves performance on a haptic spatial matching task. Exp Brain Res 149:320-330

Zuidhoek S, Visser A, Bredero ME, Postma A (2004) Multisensory integration mechanisms in haptic space perception. Exp Brain Res 157:265-268

Zuidhoek S, Kappers AML, Postma A (2007) Haptic orientation perception: sex differences and lateralization of functions. Neuropsychologia 45:332-341 